\title{
Signatures of macroscopic quantum coherence in ultracold dilute Fermi gases
}

\author{
Roberto Onofrio ${ }^{1,2,3}$ and Carlo Presilla $a^{4,3,5}$ \\ ${ }^{1}$ Department of Physics and Astronomy, Dartmouth College, \\ 6127 Wilder Laboratory, Hanover, NH 03755 \\ 2Dipartimento di Fisica "G. Galilei", \\ Università di Padova, Via Marzolo 8, Padova 35131, Italy \\ ${ }^{3}$ Center for Statistical Mechanics and Complexity, \\ INFM, Unità di Roma 1, Roma 00185, Italy \\ 4Dipartimento di Fisica, Università di Roma "La Sapienza", \\ Piazzale A. Moro 2, Roma 00185, Italy \\ ${ }^{5}$ INFN, Sezione di Roma 1, Roma 00185, Italy
}

\begin{abstract}
We propose a double-well configuration for optical trapping of ultracold two-species Fermi-Bose atomic mixtures. Two signatures of macroscopic quantum coherence attributable to a superfluid phase transition for the Fermi gas are analyzed. The first signature is based upon tunneling of Fermi pairs when the power of the deconfining laser beam is significantly reduced. The second relies on the observation of interference fringes in a regime where the fermions are trapped in two sharply separated minima of the potential. Both signatures rely on small decoherence times for the Fermi samples, which should be possible by reaching low temperatures using a Bose gas as a refrigerator, and a bichromatic optical dipole trap for confinement, with optimal heat capacity matching between the two species.

PACS numbers: 05.30.Fk, 32.80.Pj, 67.60.-g, 67.57.-z
\end{abstract}




\section{INTRODUCTION}

Degenerate Fermi gases are rather ubiquitous in nature at both the microscopic and macroscopic level, from nuclear matter to neutron stars. Studies of both their non-interacting and interacting features allow for the understanding of a wealth of physical phenomena occurring in the mesoscopic realm, in particular superconductivity. More recently, the possibility to cool dilute samples of Fermi gases below the microkelvin range has opened up a novel route to identify some of the fundamental features underlying interacting many-body Fermi systems [1]. While Pauli blocking [2] and Fermi pressure [3, 4] have been already evidenced, focus on interacting properties has recently led to interesting effects in Fermi-Bose mixtures [5], and in two-component Fermi gases [6]. In particular, in the latter case evidence has been reported for anisotropic free expansions of a Fermi cloud when this is brought to a deep degenerate regime. The data have been interpreted in terms of a superfluid state of the Fermi gas, as predicted in 7]. However, alternative interpretations in terms of hydrodynamic behaviour of a high density Fermi gas are also plausible [8]. More recently, various groups have used resonant superfluidity [9, 10, 11, 12] to explore the BEC-BCS crossover 13, 14, 15], with various claims for the formation of bound states of many body character, as expected for instance by BCS-like couplings, based on the dynamics of formation of Fermi pairs and on collective properties [16]. This reminds of the previous situation of degenerate Bose gases, when various indirect evidences were collected for the existence of a macroscopic quantum state by studying collective properties. The final evidence was only achieved by explicitely showing quantum coherence [17] and, thereafter, macroscopic quantum transport phenomena like superfluidity [18] and quantized vortices [19, 20]. Analogously, we do expect the coherence of the macroscopic wave function associated to a Cooper-paired state of Fermi atoms to play an important role to assess its superfluid nature. In this paper, we discuss a configuration for an optical dipole trap that could allow for quantitative studies of quantum coherence in an ultracold Fermi gas. In Section II we describe a geometry for an optical dipole trap which creates a bistable potential for both the Fermi species and the Bose species necessary to sympathetically cool the Fermi gas. In Section III we discuss possible signatures for macroscopic quantum coherence through tunneling phenomena in a regime where the laser intensity of the blue-detuned beam is kept low. In Section IV we describe interference experiments which should be able to disentangle between a BCS or a BEC regime 
for the degenerate Fermi gas by observing the dynamics of the fringe visibility during the free expansion of the clouds. Macroscopic coherence in itself does not rely on the Fermi gas being in an effective BEC or a BCS state, as correlated Fermi pairs, either in a molecular state (BEC limit) or a many-body state (BCS limit) always behave as quantum coherent

systems [21]. However, for the interference fringe experiment and BCS-paired fermions, a sudden loss of fringe visibility is expected for large times of flight, while such a loss is not expected in the case of fermions coupled in a molecular state. Potential decoherence sources and some technical difficulties to be overcome are then discussed in the conclusive Section $\mathrm{V}$.

\section{DOUBLE-WELL BICHROMATIC OPTICAL DIPOLE TRAPS}

The configuration we analyze relies on using an optical dipole trap made of focused reddetuned beams for trapping the atoms, and further blue-detuned beams for their selective deconfinement. Such a bichromatic optical dipole trap could allow to achieve a deep degenerate regime for a Fermi gas when the latter is sympathetically cooled through a Bose gas undergoing evaporative cooling [22].

Let us consider an optical dipole trap consisting of a single red-detuned beam (optical source 1) propagating along the $x$-axis, and a single blue-detuned beam (optical source 2), also focused on the same spot, propagating along the orthogonal axis $y$. The resulting effective potential experienced by the atoms of species $\alpha$ ( $\alpha=\mathrm{f}$ for fermions and $\alpha=\mathrm{b}$ for bosons) can be written as:

$$
\begin{array}{r}
U_{\alpha}(x, y, z)=-\frac{\hbar \Gamma_{\alpha}^{2}}{4 \pi I_{\alpha}^{\mathrm{s} a t}}\left[\frac{T_{1}^{\alpha} P_{1}}{w_{1}^{2}} \frac{\exp \left(\frac{-2\left(y^{2}+z^{2}\right)}{w_{1}^{2}\left(1+x^{2} / R_{1}^{2}\right)}\right)}{1+x^{2} / R_{1}^{2}}\right. \\
\left.+\frac{T_{2}^{\alpha} P_{2}}{w_{2}^{2}} \frac{\exp \left(\frac{-2\left(x^{2}+z^{2}\right)}{w_{2}^{2}\left(1+y^{2} / R_{2}^{2}\right)}\right)}{1+y^{2} / R_{2}^{2}}\right],
\end{array}
$$

where $T_{i}^{\alpha}=1 /\left(\Omega_{\alpha}-\Omega_{i}\right)+1 /\left(\Omega_{\alpha}+\Omega_{i}\right)$ is a parameter related to the detuning between the atomic transition angular frequencies $\Omega_{\alpha}=2 \pi c / \lambda_{\alpha}$ and the laser beam angular frequencies $\Omega_{i}=2 \pi c / \lambda_{i}\left(\lambda_{\alpha}\right.$ and $\lambda_{i}$ being the atomic transition and laser beam wavelengths, respectively), $P_{i}$ and $w_{i}$ are power and waist of the laser beams, $R_{i}=\pi w_{i}^{2} / \lambda_{i}$ their Rayleigh 
ranges, and $I_{\alpha}^{\text {sat }}=\hbar \Omega_{\alpha}^{3} \Gamma_{\alpha} / 12 \pi c^{2}$ is the saturation intensity for the atomic transition. The potential (11) is well approximated by a second order Taylor expansion around the $x$ axis

$$
U_{\alpha}(x, y, z) \simeq U_{\alpha}(\boldsymbol{x})+\frac{1}{2} \partial_{y}^{2} U_{\alpha}(\boldsymbol{x}) y^{2}+\frac{1}{2} \partial_{z}^{2} U_{\alpha}(\boldsymbol{x}) z^{2}
$$

where $\boldsymbol{x}=(x, 0,0)$ and

$$
\begin{aligned}
U_{\alpha}(\boldsymbol{x})=-\frac{\hbar \Gamma_{\alpha}^{2}}{4 \pi I_{\alpha}^{\mathrm{s} a t}}\left[\frac{T_{1}^{\alpha} P_{1}}{w_{1}^{2}} \frac{1}{1+x^{2} / R_{1}^{2}}\right. \\
\left.+\frac{T_{2}^{\alpha} P_{2}}{w_{2}^{2}} \exp \left(-2 x^{2} / w_{2}^{2}\right)\right] .
\end{aligned}
$$

The expression for $U_{\alpha}(\boldsymbol{x})$ explicitly shows that there is a soft attractive potential on the Rayleigh range scale $R_{1}$ and a sharp repulsion around the origin on the beam waist lengthscale $w_{2}$. The net effect of the beams is to establish a double well potential along the $x$-axis with minima at $\pm \boldsymbol{x}_{m}=\left( \pm x_{m}, 0,0\right)$ having at the same time a strong quasi harmonic confinement in the $y-z$ plane. The transverse angular frequencies $\omega_{\alpha y}(\boldsymbol{x})=\left(\partial_{y}^{2} U_{\alpha}(\boldsymbol{x}) / m_{\alpha}\right)^{1 / 2}$ and $\omega_{\alpha z}(\boldsymbol{x})=\left(\partial_{z}^{2} U_{\alpha}(\boldsymbol{x}) / m_{\alpha}\right)^{1 / 2}$ are one order of magnitude larger than the intra-well longitudinal angular frequency $\omega_{\alpha x}\left(\boldsymbol{x}_{m}\right)=\left(\partial_{x}^{2} U_{\alpha}\left(\boldsymbol{x}_{m}\right) / m_{\alpha}\right)^{1 / 2}$. For atomic gases with chemical potential $\mu_{\alpha}$ satisfying $\hbar \omega_{\alpha x} \ll \mu_{\alpha} \ll \hbar \omega_{\alpha y}, \hbar \omega_{\alpha z}$ the present configuration thus realizes a quasi-1D trapped gas analogously to that obtained in highly elongated magnetic traps [23].

In Fig. 11 we show the potential energy for the Fermi and Bose components in the case of the ${ }^{6} \mathrm{Li}^{2}{ }^{23} \mathrm{Na}$ mixture, already brought to degenerate regime [24], by assuming a Nd:YAG laser emitting at $\lambda_{1}=1064 \mathrm{~nm}$ as red-detuned source, and its second harmonic as bluedetuned source. Details of the potential energy profiles are shown for the two species in Fig. 2. It is evident that the bosonic species experiences a double well potential with larger distance and higher potential barrier between the minima with respect to the fermionic one.

Some comments are in order. The Fermi gas is always more deeply and strongly confined than the Bose gas, which is favorable for different reasons. Firstly, this allows for a continuous evaporative cooling of the Bose gas marginally affecting the Fermi gas. Secondly, the ratio between the average trapping frequency for fermions and bosons maintains a value greater than unity, with a negligible decrease with respect to the case of coaxial beams. This allows for efficient sympathetic cooling of a degenerate Fermi gas and a less degenerate Bose gas, through matching of the specific heats. Indeed the Bose gas, less degenerate, maintains its large classical specific heat at lower temperatures with respect to the more degenerate 


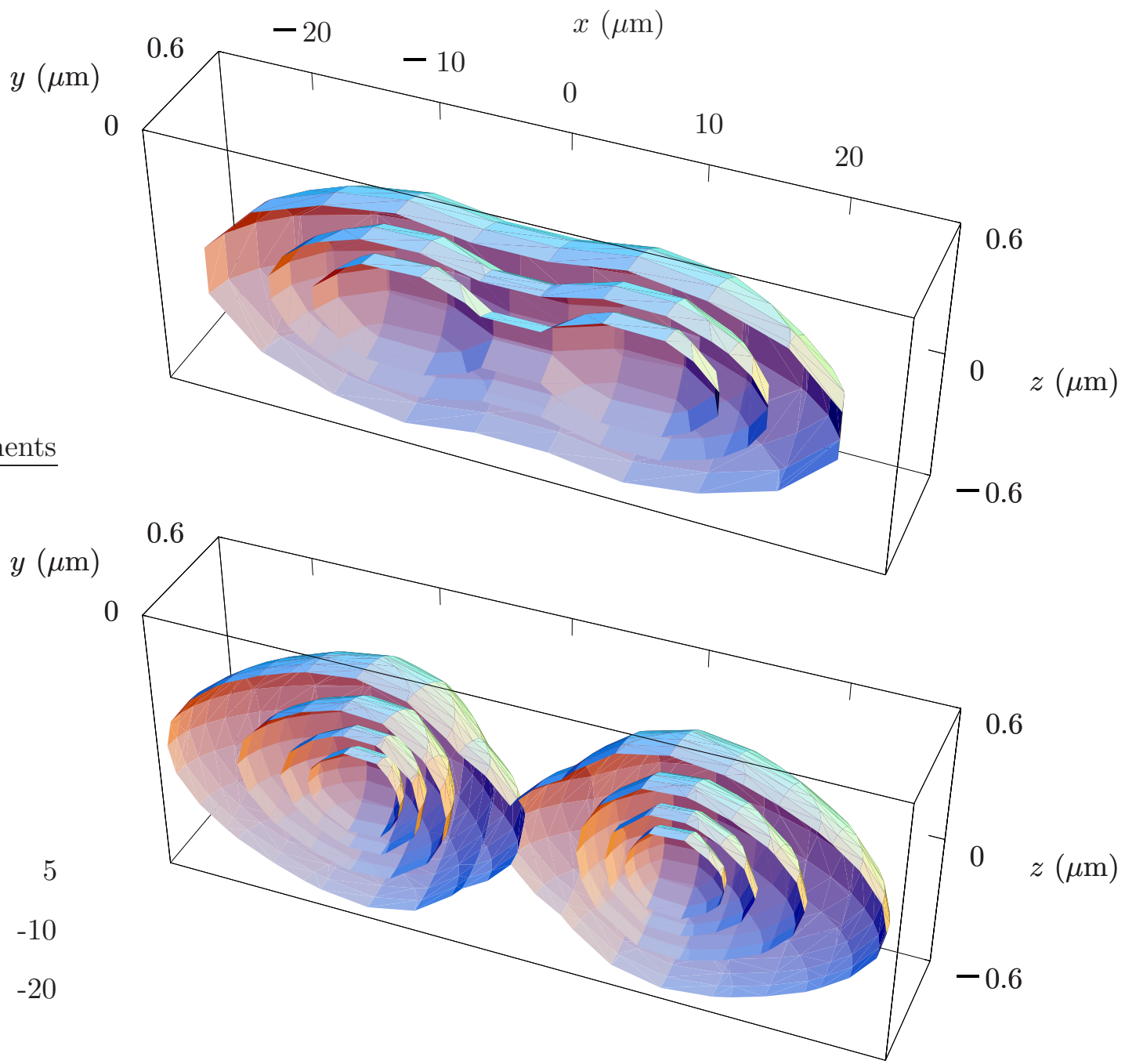

FIG. 1: Bistable potential for optically trapped Fermi-Bose mixtures. The plots represent the equipotential surfaces above the minima of the potential $U_{\alpha}^{\min }$ by an amount $\Delta U_{\alpha}=0.5,2.5,5,10$, $20 \mathrm{nK}$ (from inner to outer shells, respectively) for fermionic ${ }^{6} \mathrm{Li}$ (top) and bosonic ${ }^{23} \mathrm{Na}$ (bottom). We assume a laser power of $P_{1}=10 \mathrm{~mW}$ at $\lambda_{1}=1064 \mathrm{~nm}, P_{2} / P_{1}=2.5 \times 10^{-3}$ at $\lambda_{2}=532 \mathrm{~nm}$, and waists $w_{1}=w_{2}=10 \mu \mathrm{m}$. The atomic transition wavelengths are $\lambda_{\mathrm{f}}=671 \mathrm{~nm}$ and $\lambda_{\mathrm{b}}=589 \mathrm{~nm}$.

Fermi gas [25]. Thirdly, this reduces the peak density of the Bose gas at the center of the trap making less relevant both the boson-boson interaction, and most importantly the fermion-boson interaction.

Finally, the fact that bosons experience a higher potential barrier and a larger separation 

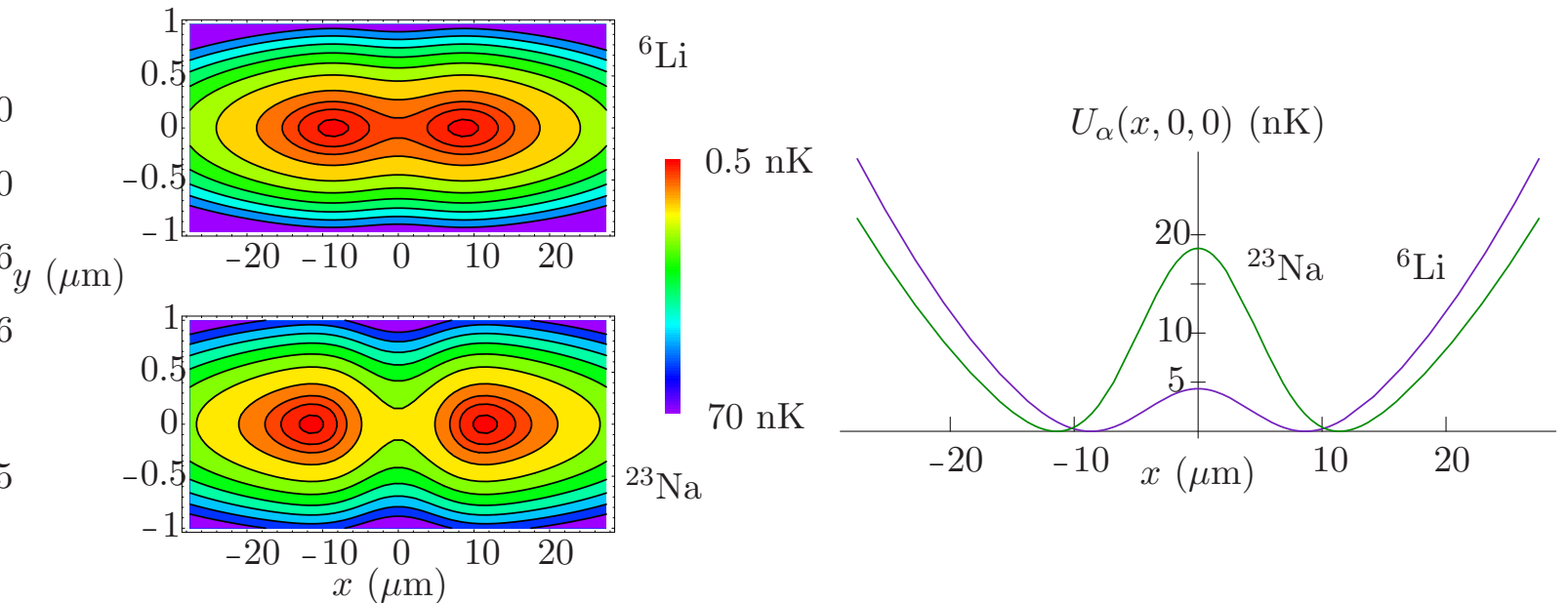

FIG. 2: Equipotential profiles in the $x-y$ plane of $U_{\alpha}(x, y, 0)$ (left), for $\Delta U_{\alpha}=0.5,2.5,5,10,20$, $30,40,50,60,70 \mathrm{nK}$ (from inner to outer shells, respectively), and potential energy along the $x$ axis for the two species (right). The minima of the bistable potentials have been shifted to zero for the sake of comparison, their values being $U_{\mathrm{f}}^{\min }=-3.87 \mu \mathrm{K}$ and $U_{\mathrm{b}}^{\min }=-3.31 \mu \mathrm{K}$. All trap parameters as in Fig. [1]

between the minima, strongly differentiates the dynamics of the two species. As explained in the following, the latter feature allows for two unambiguous signatures of the macroscopic coherence associated to a possible superfluid phase of the Fermi gas, based on tunneling oscillations and interference, respectively.

\section{TUNNELING PHENOMENA}

Macroscopic tunneling phenomena have been successfully explored in a Fermi liquid, namely ${ }^{3} \mathrm{He}$ [26], and it is therefore natural to explore their counterpart in dilute Fermi gases. In our configuration, by using sufficiently low barriers the tunneling probability for fermions may become large enough to make the detection of the corresponding interwell oscillation feasible. Here there is a rich scenario due to the possibility of single-particle tunneling for both fermions in a degenerate but normal state and bosons in a thermal state (either quantum or thermally activated), and macroscopic quantum tunneling of fermions in a BCS state and of bosons in a BEC state. An oscillating tunneling current between the two wells of the trap is obtained by breaking the symmetry along the $x$ axis by means of 
a tilting potential, $V(x)=b x$, suddenly added to the trapping potential $U_{\alpha}(x, y, z)$, e.g. using Zeeman shifts generated by a quadrupole magnetic field with symmetry axis along the $x$ direction.

The evaluation of the macroscopic tunneling current is particularly simple within the quasi one-dimensional approximation (2) of the potential. Suppose that the total number $N_{\alpha}$ of trapped (bosons or bosonized fermions) atoms avaliable for macroscopic tunneling is in the ground state of the potential (3) and let $\psi_{\alpha}(x)$ be the unit-normalized associated wavefunction. If a tilting potential $V(x)=b x$ with small bias parameter $b$ is added at time $t=0$, the evolution of the system wavefunction can be obtained as

$$
\psi_{\alpha}(x, t)=c_{1}^{\alpha} e^{i E_{1}^{\alpha} t / \hbar} \phi_{1}^{\alpha}(x)+c_{2}^{\alpha} e^{i E_{2}^{\alpha} t / \hbar} \phi_{2}^{\alpha}(x),
$$

where $\phi_{1}^{\alpha}(x)$ and $\phi_{2}^{\alpha}(x)$ are the first two eigenfunctions, with eigenvalues $E_{1}^{\alpha}$ and $E_{2}^{\alpha}$, respectively, of the tilted potential $U_{\alpha}(\boldsymbol{x})+V(x)$ and the coefficients $c_{1}^{\alpha}$ and $c_{2}^{\alpha}$ are given by

$$
c_{i}^{\alpha}=\int_{-\infty}^{+\infty} \phi_{i}^{\alpha}(x) \psi_{\alpha}(x) d x, \quad i=1,2
$$

In the absence of decoherence phenomena the evolution of the macroscopic wavefunction (4) gives rise to condensate fractions in the left and right wells, $N_{\alpha}^{L}(t)$ and $N_{\alpha}^{R}(t)$ oscillating in time with $N_{\alpha}^{L}(t)+N_{\alpha}^{R}(t)=N_{\alpha}$. The corresponding current is easily evaluated as

$$
\frac{d N_{\alpha}^{L}}{d t}=N_{\alpha} \frac{d}{d t} \int_{-\infty}^{0}\left|\psi_{\alpha}(x, t)\right|^{2} d x=A_{\alpha} \sin \frac{\Delta E_{\alpha} t}{\hbar}
$$

with $A_{\alpha}=2 c_{1}^{\alpha} c_{2}^{\alpha} c_{12}^{\alpha} N_{\alpha} \Delta E_{\alpha} / \hbar$ the amplitude of the tunneling current, depending upon the energy splitting $\Delta E_{\alpha}=E_{2}^{\alpha}-E_{1}^{\alpha}$, and

$$
c_{12}^{\alpha}=\int_{-\infty}^{0} \phi_{1}^{\alpha}(x) \phi_{2}^{\alpha}(x) d x
$$

is the overlap integral.

To evaluate the macroscopic tunneling current for the realistic trap potential (2), the eigenvalues $E_{1}^{\alpha}$ and $E_{2}^{\alpha}$ and the corresponding eigenfunctions $\phi_{1}^{\alpha}$ and $\phi_{2}^{\alpha}$ must be found numerically. Due to the strongly differentiated tunneling dynamics for bosons and bosonized fermions, we have the hard numerical problem of finding exceedingly small energy splittings. The selective relaxation algorithm proposed in [27] allows to solve the problem at least in the parameter region of physical interest. 


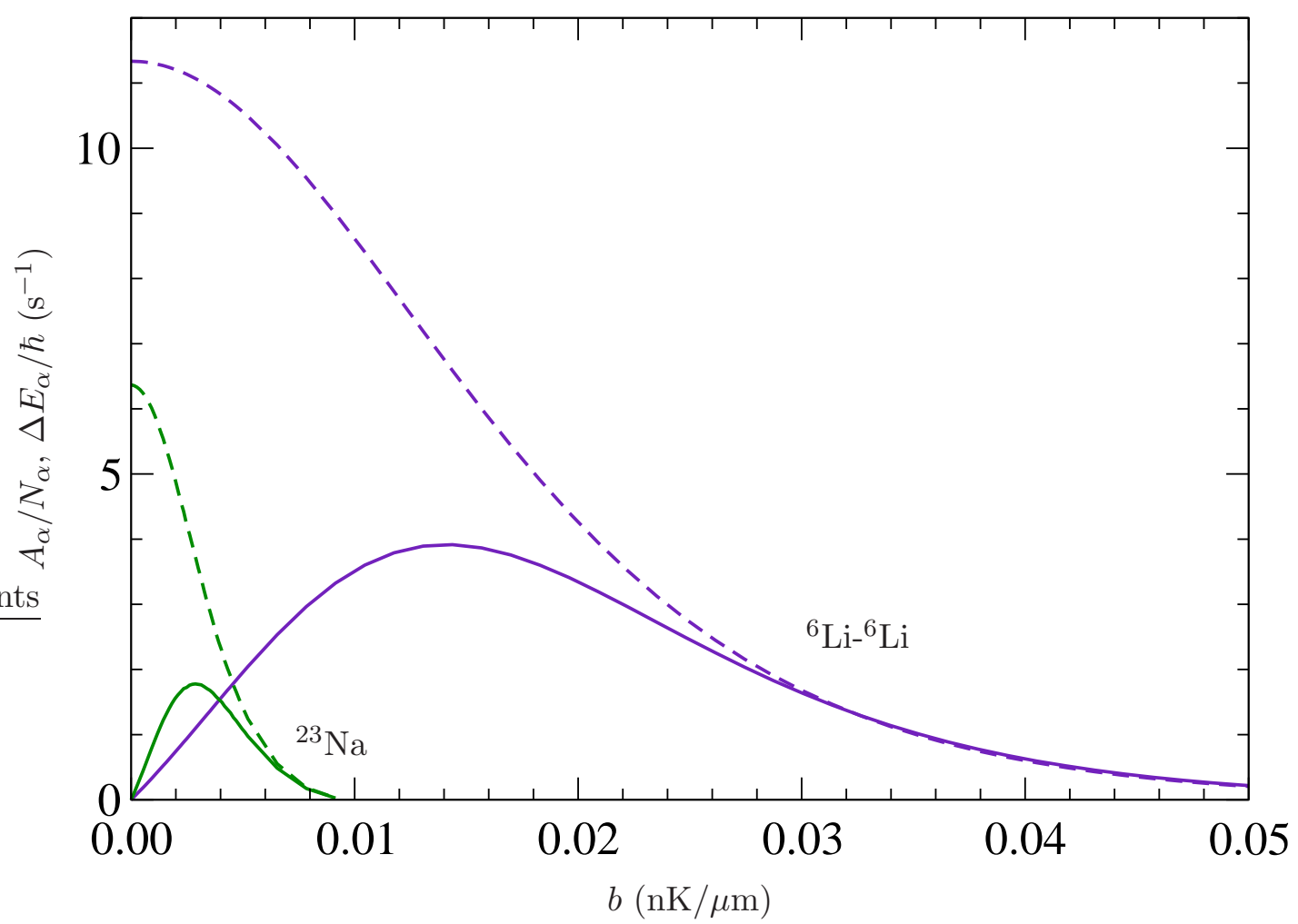

FIG. 3: Macroscopic coherence of the Fermi gas through tunneling experiments. Dependence of tunneling current amplitude per unit of available atoms $A_{\alpha} / N_{\alpha}$ (solid line) and tunneling angular frequency $\Delta E_{\alpha} / \hbar$ (dashed line) for ${ }^{23} \mathrm{Na}$ and ${ }^{6} \mathrm{Li}^{6}{ }^{6} \mathrm{Li}$ Cooper pairs versus bias strength $b$. For the sake of comparison, amplitude and frequency of bosons have been multiplied by $10^{5}$. The trap parameters are as in Fig. 1]

In Fig. 3 we report the amplitude and the angular frequency of the currents for macroscopic tunneling of ${ }^{6} \mathrm{Li}^{-}{ }^{6} \mathrm{Li}$ Cooper pairs and of the Bose condensed component of ${ }^{23} \mathrm{Na}$ versus the tilting parameter $b$. For both species there is an optimum value of the bias maximizing the observability of tunneling oscillations, with a maximum value $A_{\alpha} \simeq 4 \mathrm{~s}^{-1}$ obtained for a bias value of the tilting potential $b \simeq 14 \mathrm{pK} / \mu \mathrm{m}$. Tunneling current and frequency for the Bose gas are smaller by five orders of magnitude with respect to the analogous quantities of the Cooper pair gas. This results from the exponential sensitivity of quantum tunneling to the different potentials experienced by the two species and to their different masses.

Provided that the number of Cooper pairs is large enough, the modulation of the number of atoms in the two wells can be evidenced by using non-destructive phase-contrast imaging. The low intensity of the blue-detuned beam required to create a tunneling regime in the presence of a magnetic trap with limited confinement strength prevented observation of 
tunneling phenomena in the experiment described in [17] (see also [28] for a recently achieved bistable configuration). In the situation proposed here this issue is circumvented due to the possibility to change both the intensities of the beams while maintaining their ratio constant, at least to the extent that heating from residual Rayleigh scattering does not play a significant role. Current techniques allow for relative stabilization at the $1 \%$ level or below (for a general discussion of laser stabilization techniques see [29]), especially for frequency-doubled beams as in the proposed configuration.

An important requirement for this proposed test is to maintain the decoherence rate low enough to minimize damping of the coherent oscillations which, according to Fig. 3, are expected to occur with periods of order $100 \mathrm{~ms}$ or longer. In the cooling strategy outlined in 25] there is not a strict need to use enhancement of the elastic scattering length through Feshbach resonances [9, 10] to reach a deep Fermi degenerate regime. This could circumvent the issue of decoherence sources due to enhancement of density, like those discussed in 30] for three-body collisions. Thus decoherence is mainly expected from the presence of Rayleigh heating or more technical sources like intensity and beam pointing fluctuations of the laser beams. Their effect is a temperature increase with a consequent increase of the thermal component [31] and the single-particle tunneling current of bosons and fermions. It should be noted however that in an optical dipole trap, due to the smaller trapping volume and lower trap depth, we expect a suppressed thermal fraction. This is a further advantage in using an optical trap with respect to a magnetic trap for coherence experiments. Continuous evaporative cooling of the Bose species should also mitigate its effect. One can then envisage a cooling strategy where coherent oscillations persist for a much longer time at time-dependent amplitude and frequency, therefore originating a chirped signal.

\section{INTERFERENCE PHENOMENA}

In a landmark experiment, the Ketterle group evidenced the macroscopic coherence of a pair of Bose condensates by looking at the interference fringes resulting after their release from a bistable potential [17]. The latter was obtained by the combination of a harmonic potential created by a magnetic trap, and a blue-detuned beam focused on the magnetic potential minimum with propagation orthogonal to the weaker confining axis. The average distance between the two condensates was controlled by changing the power of the blue- 
detuned beam.

A similar experiment can be repeated with an ultracold Fermi-Bose mixture in a bistable optical dipole trap. Above the temperature for the onset of a BCS-like phase transition of the Fermi gas one expects only interference fringes arising from the condensed fraction of the Bose gas. Below $T_{\mathrm{BCS}}$ we do expect also the emergence of an interference pattern coming from the bosonized fermions, a small fraction of the total number of Fermi atoms. The distance between the peaks of maximum signal in the interference pattern is given, for a free expansion, by $\ell_{\alpha}=2 \pi \hbar t / m_{\alpha} d_{\alpha}$. Here $t$ is the time of flight, $d_{\alpha}$ the initial distance between the centers of mass of the two clouds before the release from the trap, and $m_{\alpha}$ either the mass of the Bose atoms or twice the mass of each fermion in the case of the BCS-bosonized component. Since the Fermi species in our example has both a significantly smaller mass and separation $d_{\alpha}$ than the Bose species we do expect an easy discrimination of the interference pattern attributable to the former species. This is confirmed by looking at the distance between the interference peaks for ${ }^{6} \mathrm{Li}$ and ${ }^{23} \mathrm{Na}$ versus the $P_{2} / P_{1}$ power ratio, as depicted in Fig. 4. One can take advantage of this dependence of the interference patterns to discriminate any effect of the Bose component. The spacing of the interference fringes expected from the macroscopic wavefunction associated to the bosonized Fermi component maintains a value $\sim 2$ times larger than the corresponding one for the Bose species. Selective optical pumping tomography on the Fermi species cycling transition as in [17] can allow to enhance the corresponding interference signal. Considering the very dilute nature of the bosonized Fermi gas, as a consequence of the less stiff confinement of the Bose species, we do not expect a significant decrease of the fringe visibility due to the mean field effects as instead already observed for bosons [32]. Due to the lower trapping frequencies when using a bichromatic optical trap as discussed in 22], the mean-field effects due to the interaction between the Fermi and the Bose gas are also negligible.

The interference experiment discussed above could also allow to distinguish if the Fermi gas is in a BCS or BEC regime. For BCS macroscopic states we do expect a loss of fringe contrast at expansion times much smaller than those reachable in the case of molecular BEC states. This behaviour is quantitatively shown by assuming an adiabatic expansion of the gas after the release from the trap, and a rough estimate of the critical temperatures, $T_{\mathrm{BCS}}$ and $T_{\mathrm{BEC}}$ as follows. Suppose that at time $t=0$, when the trap potential is turned off, the gas made of $N_{\mathrm{F}}$ fermions of mass $m_{\mathrm{F}}$ has density $n_{0}$. Let $R_{0}$ be the radius of the 


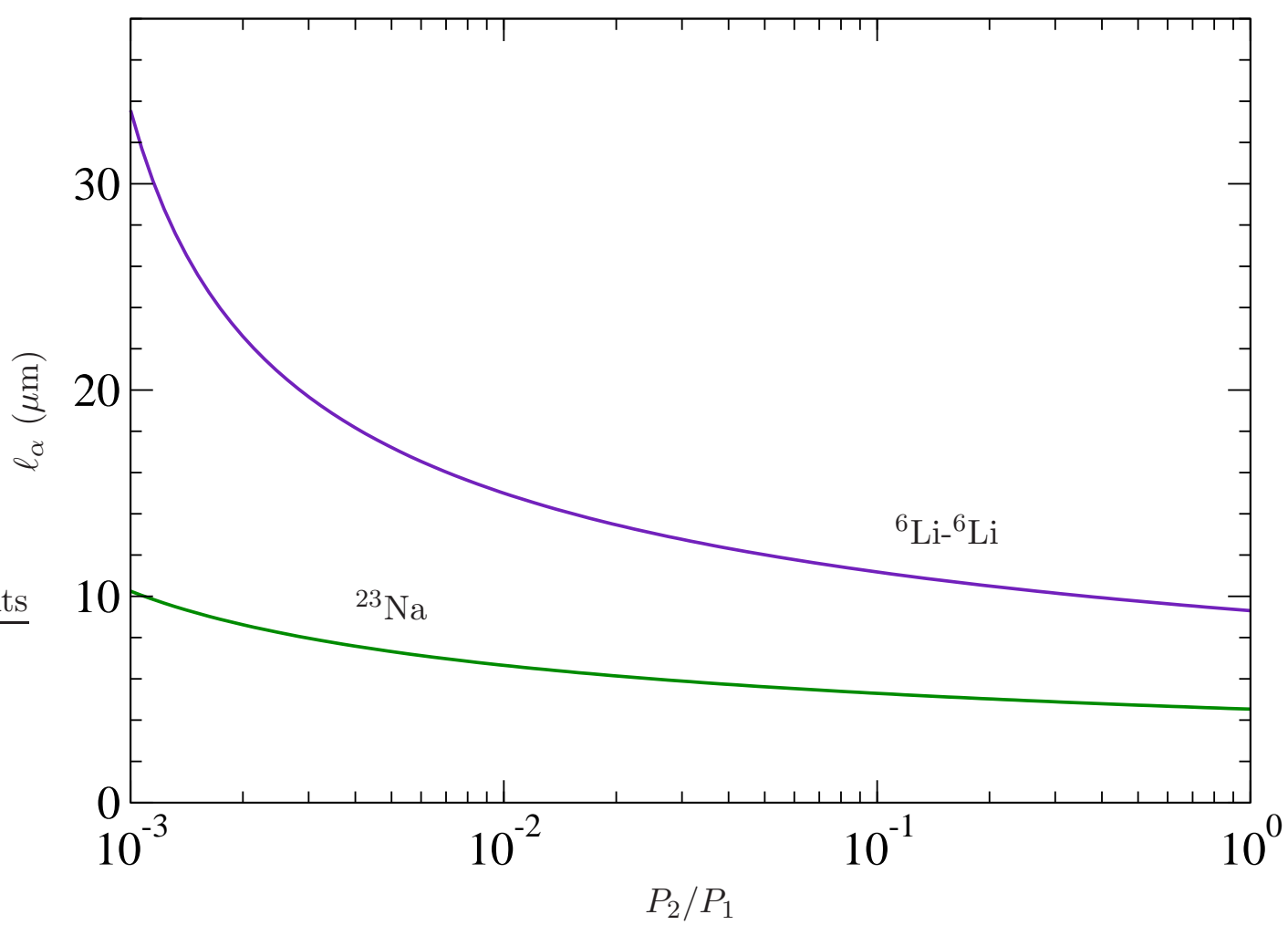

FIG. 4: Macroscopic coherence of the Fermi gas through interference experiments. Dependence of fringe spacing $\ell_{\alpha}$ for ${ }^{23} \mathrm{Na}$ bosons and ${ }^{6} \mathrm{Li}^{6}{ }^{6} \mathrm{Li}$ Cooper pairs versus $P_{2} / P_{1}$. We assume a time of flight $t=10 \mathrm{~ms}$.

equivalent sphere containing the gas at $t=0$ defined by $\frac{4}{3} \pi R_{0}^{3} n_{0}=N_{\mathrm{F}}$. At later times the cloud expands and, assuming an ideal behavior, the radius of the equivalent sphere is given by the law $R(t)=R_{0}+v_{\mathrm{F}} t$. As a consequence, the density of the gas $n(t)$ decreases from its initial value $n_{0}$ and, in turn, the Fermi velocity $v_{\mathrm{F}}=\hbar\left(3 \pi^{2} n\right)^{1 / 3} / m_{\mathrm{F}}$ also decreases. The density of the gas is therefore determined by the following self-consistent equation

$$
n(t)=\frac{N_{\mathrm{F}}}{\frac{4}{3} \pi\left[R_{0}+\frac{\hbar t}{m_{\mathrm{F}}}\left(3 \pi^{2} n(t)\right)^{\frac{1}{3}}\right]^{3}},
$$

which, for a generic value of $t$, must be solved numerically. For $t \gg t_{0}=m_{\mathrm{F}} R_{0} / \hbar\left(3 \pi^{2} n_{0}\right)^{1 / 3}$, the initial radius $R_{0}$ can be neglected in the denominator of (8) and we obtain the asymptotic time-varying density

$$
n_{\infty}(t)=\sqrt{\frac{n_{0}}{3 \pi^{2}}\left(\frac{m_{\mathrm{F}} R_{0}}{\hbar t}\right)^{3}} .
$$

Once the density $n(t)$ is known, we have an explicit estimate of the critical temperature for 


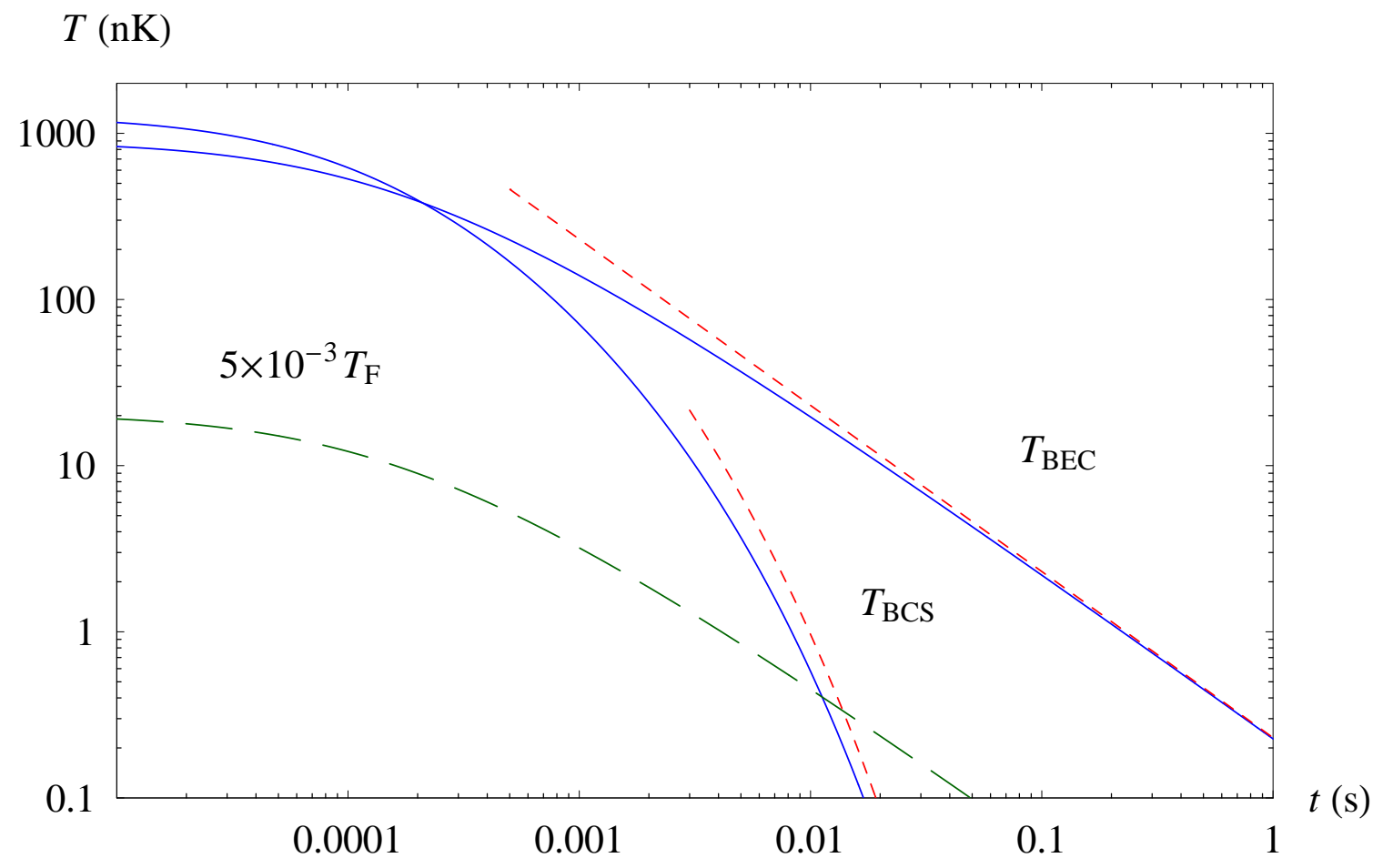

FIG. 5: Temperature of the cloud (dashed line), and critical temperatures $T_{\mathrm{BCS}}, T_{\mathrm{BEC}}$ (continuous lines) as a function of time $t$ in the case of a freely expanding ${ }^{6} \mathrm{Li}$ cloud with $N_{\mathrm{F}}=3 \times 10^{6}$ atoms. The initial density is $n_{0}=3.5 \times 10^{13} \mathrm{~cm}^{-3}$ and we use an elastic scattering length for fermions $a=-230 \mathrm{~nm}$ [1]. The dashed lines close to the critical temperature continuous lines are the asymptotic values for $T_{\mathrm{BCS}}, T_{\mathrm{BEC}}$ obtained by substituting $n(t)$ with $n_{\infty}(t)$ into (10) and (11).

BCS transition [1] as a function of time

$$
T_{\mathrm{BCS}}(t)=\frac{5}{3 e} \frac{\hbar^{2}\left(3 \pi^{2} n(t)\right)^{\frac{2}{3}}}{2 m_{\mathrm{F}} k_{\mathrm{B}}} \exp \left[-\frac{\pi}{2|a|(3 \pi n(t))^{\frac{1}{3}}}\right],
$$

where $a$ is the elastic scattering length of the fermionic species. The critical temperature $T_{\mathrm{BEC}}$ for fermions condensed via molecular states can be estimated from the critical temperature of an ideal gas of bosons [33] with mass $2 m_{\mathrm{F}}$ and density $n(t) / 2$

$$
T_{\mathrm{BEC}}(t)=\frac{\pi \hbar^{2}}{m_{\mathrm{F}} k_{\mathrm{B}}}\left(\frac{n(t)}{2 \zeta(3 / 2)}\right)^{2 / 3}
$$

where $\zeta$ is the Riemann zeta function.

In Fig. 5 we show the behavior of the critical temperatures $T_{\mathrm{BCS}}$ and $T_{\mathrm{BEC}}$, and of the temperature of the cloud assuming an adiabatic expansion [7], versus time in the case of ${ }^{6} \mathrm{Li}$. While $T_{\mathrm{BEC}}$ has only a $n(t)^{2 / 3}$ dependence, and therefore decreases for large times as $t^{-1}$, 
$T_{\mathrm{BCS}}$ has a further exponential suppression factor and for large $t$ decreases as $t^{-1} \exp (-\sqrt{t})$. By assuming an initial temperature $T=5 \times 10^{-3} T_{\mathrm{F}}$, the disappearance of the fringes at times $t \sim 10 \mathrm{~ms}$ when $T / T_{\mathrm{BCS}}>1$ would imply a BCS regime for the degenerate Fermi gas. On the other hand, the observation of interference effects should be possible up to longer times in the case of fermions coupled through molecular BEC, since the scaling of the temperature of the cloud and $T_{\mathrm{BEC}}$ are similar.

\section{CONCLUSIONS}

We have discussed a quasi one-dimensional bistable configuration for optically trapped atoms. Two signatures have been discussed for evidencing macroscopic quantum coherence of a paired component of a Fermi gas, regardless of the BEC or BCS-like regime for the Fermi gas. Our proposal allows to identify a superfluid component both in a strongly-coupled regime based upon enhancement of scattering length (molecular BEC regime) or in a BCSlike regime obtained by just cooling the sample at very low temperatures as suggested in 25], with efficient heat capacity matching between the Fermi and the Bose species, without necessarily exploiting Feshbach resonances to obtain large critical temperatures for BCS pairing, although their use is certainly possible in our framework. The use of a Fermi-Bose mixture seems preferable since analogous quantum coherence experiments involving mixtures of two Zeeman levels of fermions are less easy to perform. Indeed, two distinguishable Fermi states with same mass will give rise to two independent interference patterns or tunneling currents just differing by the initial random phase, unless a locking mechanism is used. Also, dual evaporative cooling substantially decreases the number of atoms potentially available for Cooper pairing, then sensibly diminishing the expected signal. Moreover, the presence of the Bose gas until the last stage of the cooling is useful to quantitatively assess the temperature of the Fermi gas, and allows to study a variety of situations for which changes to the BCS-coupled Fermi pairs are expected when bosons mediates their interactions [34].

There have been many refined and ingenious proposals for the observation of the superfluid phase of an ultracold Fermi gas, ranging from the study of collective modes [35], moment of inertia 36], density profile of the Cooper-paired component [1], light scattering 37, 38, 39, 40, 41, 42], free expansion [7], Bloch oscillations 43], internal Josephson effect 44], and Raman photoassociation in Bose-Fermi mixtures [45]. All these proposals, includ- 
ing ours, will have to face the small number of fermions available in the superfluid state, and the subsequent small signal-to-noise ratio for any conceivable signature of the phase transition. In view of these experimental difficulties it is crucial to seek for redundancy of signatures with diverse techniques, hopefully all converging in individuating a common superfluid phase diagram.

\section{Acknowledgments}

We thank M. L. Chiofalo for useful discussions, and L. Viola for a critical reading of the manuscript. This work was supported in part by Cofinanziamento MIUR protocollo 2002027798_001.

[1] H. T. C. Stoof, M. Houbiers, C. A. Sackett, and R. G. Hulet, Phys. Rev. Lett. 76, 10 (1996).

[2] B. DeMarco and D. S. Jin, Science 285, 1703 (1999).

[3] A. G. Truscott, K. E. Strecker, W. I. McAlexander, G. B. Partridge, and R. G. Hulet, Science 291, 2570 (2001).

[4] F. Schreck, L. Khaykovich, K. L. Corwin, G. Ferrari, T. Bourdel, J. Cubizolles, and C. Salomon, Phys. Rev. Lett. 87, 080403 (2001).

[5] G. Modugno, G. Roati, F. Riboli, F. Ferlaino, R. J. Brecha, and M. Inguscio, Science 297, 2240 (2002).

[6] K. M. O'Hara, S. L. Hemmer, M. E. Gehm, S. R. Granade, and J. E. Thomas, Science 298, 2179 (2002).

[7] C. Menotti, P. Pedri, and S. Stringari, Phys. Rev. Lett. 89, 250402 (2002).

[8] S. Gupta, Z. Hadzibabic, J. R. Anglin, and W. Ketterle, Phys. Rev. Lett. 92, 100401 (2004).

[9] M. Holland, S.J.J.M.F. Kokkelmans, M. L. Chiofalo, and R. Walser, Phys. Rev. Lett. 87, 120406 (2001).

[10] E. Timmermans, V. Furuya, P. W. Milonni, and A. K. Kerman, Phys. Lett. A 285, 228 (2001).

[11] M.L. Chiofalo, S.J.J.M.F. Kokkelmans, J. N. Milstein, and M. J. Holland, Phys. Rev. Lett. 88, 090402 (2002).

[12] Y. Ohashi and A. Griffin, Phys. Rev. Lett. 89, 130402 (2002). 
[13] C. A. Regal, M. Greiner, and D. S. Jin, Phys. Rev. Lett. 92, 040403 (2004).

[14] M. Bartenstein, A. Almeyer, S. Riedl, S. Jochim, C. Chin, J. H. Denschlag, and R. Grimm, Phys. Rev. Lett. 92, 120401 (2004).

[15] M. W. Zwierlein, C. A. Stan, C. H. Schunck, S. M. F. Raupach, A. J. Kerman, and W. Ketterle, Phys. Rev. Lett. 92, 120403 (2004).

[16] J. Kinast, S. L. Hemmer, M. E. Gehm, A. Turlapov, and J. E. Thomas, Phys. Rev. Lett. 92, $150402(2004)$.

[17] M. R. Andrews, C. G. Townsend, H.-J. Miesner, D. S. Durfee, D. M. Kurn, and W. Ketterle, Science 275, 637 (1997).

[18] C. Raman, M. Köhl, R. Onofrio, D. S. Durfee, C. E. Kuklewicz, Z. Hadzibabic, and W. Ketterle, Phys. Rev. Lett. 83, 2502 (1999); O. M. Maragó, S. A. Hopkins, J. Arlt, E. Hodby, G. Hechenblaikner, and C. J. Foot, Phys. Rev. Lett. 84, 2056 (2000); R. Onofrio, C. Raman, J. M. Vogels, J. R. Abo-Shaeer, A. P. Chikkatur, and W. Ketterle, Phys. Rev. Lett. 85, 2228 (2000).

[19] M. R. Matthews, B. P. Anderson, P. C. Haljan, D. S. Hall, C. E. Wieman, and E. A. Cornell, Phys. Rev. Lett. 83, 2498 (1999); K. W. Madison, F. Chevy, W. Wohlleben, J. Dalibard, Phys. Rev. Lett. 84, 806 (2000); J. R. Abo-Shaeer, C. Raman, J. M. Vogels, and W. Ketterle, Science 292, 476 (2001).

[20] A. L. Fetter and A. A. Svidzinsky, J. Phys. Cond. Matter 13, R 135 (2001).

[21] D. G. McDonald, Physics Today 54 (7), 46 (2001).

[22] R. Onofrio and C. Presilla, Phys. Rev. Lett. 89, 100401 (2002); J. Stat. Phys. 115, 57 (2004).

[23] A. Görlitz, J. M. Vogels, A. E. Leanhardt, C. Raman, T. L. Gustavson, J. R. Abo-Shaeer, A. P. Chikkatur, S. Gupta, S. Inouye, T. Rosenband, and W. Ketterle, Phys. Rev. Lett. 87, $130402(2001)$.

[24] Z. Hadzibabic, C. A. Stan, K. Dieckmann, S. Gupta, M. W. Zwierlein, A. Görlitz, and W. Ketterle, Phys. Rev. Lett. 88, 160401 (2002); Z. Hadzibabic, S. Gupta, C. A. Stan, C. H. Schunck, M. W. Zwierlein, K. Dieckmann, and W. Ketterle, Phys. Rev. Lett. 91, 160401 (2003).

[25] C. Presilla and R. Onofrio, Phys. Rev. Lett. 90, 030404 (2003).

[26] O. Avenel and E. Varoquaux, Phys. Rev. Lett. 60, 416 (1988); J. C. Davis and R. E. Packard, Rev. Mod. Phys. 74, 741 (2002). 
[27] C. Presilla and U. Tambini, Phys. Rev. E 52, 4495 (1995).

[28] Y. Shin, M. Saba, A. Schirotzek, T. A. Pasquini, A. E. Leanhardt, D. E. Pritchard, and W. Ketterle, Phys. Rev. Lett. 92, 150401 (2003).

[29] T. C. Ralph, E. H. Huntington, C. C. Harb, B. C. Buchler, P. K. Lam, D. E. McClelland, and H.-A. Bachor, Opt. Quant. Electr. 31, 583 (1999).

[30] M. W. Jack, Phys. Rev. Lett. 89, 140402 (2002).

[31] R. Graham, Phys. Rev. Lett. 81, 5262 (1998).

[32] A. Röhrl, M. Naraschewski, A. Schenzle, and H. Wallis, Phys. Rev. Lett. 78, 4143 (1997).

[33] F. Dalfovo, S. Giorgini, L. P. Pitaevskii, and S. Stringari, Rev. Mod. Phys. 71, 463 (1999).

[34] H. Heiselberg, C. J. Pethick, H. Smith, and L. Viverit, Phys. Rev. Lett. 85, 2418 (2000); L. Viverit, C. J. Pethick, and H. Smith, Phys. Rev. A 61, 053605; M. J. Bijlsma, B. A. Heringa, and H. T. C. Stoof, Phys. Rev. A 61, 053601 (2000); D. V. Efremov and L. Viverit, Phys. Rev. B 65, 134519 (2002); L. Viverit, Phys. Rev. A 66, 023605 (2002).

[35] M. A. Baranov and D. S. Petrov, Phys. Rev. A 62, 041601 (2000); A. Minguzzi and M. P. Tosi, Phys. Rev. A 63, 023609 (2001).

[36] M. Farine, P. Schuck, and X. Viñas, Phys. Rev. A 62, 013608 (2000); M. Urban and P. Schuck, Phys. Rev. A 67, 033611 (2003).

[37] W. Zhang, C. A. Sackett, and R. G. Hulet, Phys. Rev. A 60, 504 (1999).

[38] P. Törma and P. Zoller, Phys. Rev. Lett. 85, 487 (2000).

[39] J. Ruostekoski, Phys. Rev. A 61, 033605 (2000).

[40] F. Weig and W. Zwerger, Europhys. Lett. 49, 282 (2000).

[41] T. Wong, Ö. Müstecaplioglu, L. You, and M. Lewenstein, Phys. Rev. A 62, 033608 (2000).

[42] C. P. Search, H. Pu, W. Zhang, and P. Meystre, Phys. Rev. Lett. 88, 110401 (2002).

[43] M. Rodriguez and P. Törma, Phys. Rev. A 69, 041602(R) (2003).

[44] Gh.-S. Paraoanu, M. Rodriguez, and P. Törma, Phys. Rev. A 66, 041603(R) (2002).

[45] M. Mackie, O. Dannenberg, J. Piilo, K.-A. Suominen, and J. Javanainen, Phys. Rev. A 69, $053614(2004)$. 